\title{
OUTFLOWS FROM DYNAMO-ACTIVE PROTOSTELLAR ACCRETION DISCS
}

\author{
BRIGITTA VON REKOWSKI ${ }^{1}$, AXEL BRANDENBURG $^{2}$, WOLFGANG DOBLER $^{3}$ \\ and ANVAR SHUKUROV ${ }^{4}$ \\ ${ }^{1}$ Department of Astronomy and Space Physics, Uppsala University, Uppsala, Sweden; \\ E-mail:Brigitta.vonRekowski@astro.uu.se \\ ${ }^{2}$ NORDITA, Copenhagen, Denmark \\ ${ }^{3}$ Kiepenheuer-Institut für Sonnenphysik, Freiburg, Germany \\ ${ }^{4}$ School of Mathematics and Statistics, University of Newcastle, Newcastle, UK
}

\begin{abstract}
An axisymmetric model of a cool, dynamo-active accretion disc is applied to protostellar discs. Thermally and magnetically driven outflows develop that are not collimated within $0.1 \mathrm{AU}$. In the presence of a central magnetic field from the protostar, accretion onto the protostar is highly episodic, which is in agreement with earlier work.
\end{abstract}

Keywords: protostellar discs, outflows, magnetospheric accretion

\section{Introduction}

Outflows from protostellar discs have been observed in many star-forming regions. These outflows are generally believed to be driven by magneto-centrifugal acceleration from the accretion disc around the protostar. Yet, most theoretical approaches to understanding stellar outflows have ignored or at least greatly oversimplified both accretion disc physics and magnetic field evolution. Instead, most effort so far has focused around the physics of magnetised outflows and the collimation process assuming a fixed magnetic field. One of the perhaps most convincing simulations of the collimation of outflows have come from Ouyed et al. (1997) and Ouyed and Pudritz (1997) who considered a disc corona with an imposed field aligned with the rotation axis of the disc and modelled the disc physics as a boundary condition.

In recent years several groups have begun to approach the problem from different directions. One approach is to start from accretion disc physics and to extend the simulation domain in the vertical direction, so as to capture parts of the outflow acceleration region. The global simulations of Hawley (2000) are among the most impressive attempts to treat the problem as a whole. Here the magnetic field is generated by the disc dynamo driven by the magneto-rotational instability and some form of outflow is indeed seen in the vicinity of the rotation axis. However, the dynamo still seems to be unable to produce a large scale poloidal field (which seems to be required for driving systematic outflows), and thermodynamics is not included to allow the disc to cool sufficiently to become geometrically thin. Other groups have focused on the dynamo aspect and have used thin disc theory as input to

Astrophysics and Space Science 292: 493-500, 2004.

(c) 2004 Kluwer Academic Publishers. Printed in the Netherlands. 
their models (Reyes-Ruiz and Stepinski, 1995) or have studied the effect of vertical outflows on the disc dynamo (Bardou et al., 2001).

In the present paper we review the approach of von Rekowski et al. (2003) which is conceptually closest to the dynamo approach mentioned above. The effect of the Lorentz force on the gas motions is taken into account. For a thin accretion disc, it is required that near-Keplerian shear velocity be the result of a balance mainly between centrifugal force and gravity. This is only possible if the disc is cool, and remains cool notwithstanding the adiabatic heating associated with the concentration of gas near the midplane by the vertical component of gravity during the disc formation.

In order to stay conceptually as close as possible to the polytropic setup of Ouyed and Pudritz (1997) we adopt a bi-polytropic model with low entropy in the disc and high entropy in the exterior which we refer to as halo or corona (see the next section).

Since we restrict ourselves to two-dimensional geometry, we model the disc dynamo using mean-field ( $\alpha \Omega$ dynamo) theory. The applicability of this theory to protostellar accretion discs, where the turbulence (and the dynamo) is driven by the magneto-rotational (or Balbus-Hawley) instability, will also be discussed in the next section.

\section{Modelling a Cool Dynamo-Active Accretion Disc}

Like Ouyed and Pudritz (1997) we start with an equilibrium corona, where we assume constant entropy and hydrostatic equilibrium according to $c_{p} T(r)=G M_{*} / r$. (Here, $T$ is temperature, $G$ is the gravitational constant, $M_{*}$ is the mass of the protostar, $r$ is the spherical radius, and $c_{p}$ is the specific heat at constant gas pressure.) In order to make the disc cooler, we prescribe a geometrical region for the disc (see Figure 1) and choose an entropy contrast between disc and corona such that the initial disc temperature is about $3 \times 10^{3} \mathrm{~K}$ in the bulk of the disc. Given the degree of simplification of our model, we have refrained from modelling the flaring

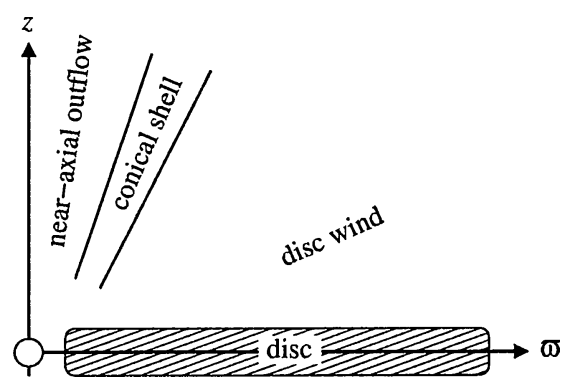

Figure 1. General structure of the outflows typically obtained in our model. The cool, dense disc emits (a) a thermally driven wind (slow, hot, dense, magnetised, rotating), (b) a magneto-centrifugally driven outflow in a conical shell (faster, cooler, less dense, magnetised, quickly rotating), and (c) a thermally driven outflow near the axis (slow, hot, dense, weakly magnetised and weakly rotating). 
of the disc. The low disc temperature corresponds to a high disc density of about $10^{-10} \ldots 10^{-9} \mathrm{~g} \mathrm{~cm}^{-3}$.

Hydrostatic equilibrium requires $(\boldsymbol{u} \cdot \nabla) \boldsymbol{u}=-(1 / \rho) \nabla p-\nabla \Phi$, where $\boldsymbol{u}=$ $\left(0, u_{\varphi}, 0\right)$ is the velocity field, $p$ is gas pressure, $\rho$ is gas density, and $\Phi$ is the gravitational potential. Since specific entropy $s$ is given, it is convenient to express $(1 / \rho) \nabla p$ in terms of $s$ and specific enthalpy $h$, using the relation $\nabla h=(1 / \rho) \nabla p+$ $T \nabla s$ (first law of thermodynamics) and $h=c_{p} T$. We obtain $h$ by integrating the vertical balance equation,

$$
-\partial(h+\Phi) / \partial z+h \partial\left(s / c_{p}\right) / \partial z=0,
$$

from large $z$ (where $h+\Phi=0$ ) down to $z=0$. The initial rotation velocity, $u_{\varphi 0}$, follows from the radial balance equation,

$$
-u_{\varphi 0}^{2} / \varpi=-\partial(h+\Phi) / \partial \varpi+h \partial\left(s / c_{p}\right) / \partial \varpi .
$$

In the disc, $h=c_{p} T$ is small, so $u_{\varphi 0}$ is slightly sub-Keplerian, while the corona does not rotate initially, and is supported by the gas-pressure gradient. Within the disc, we adjust density with a forcing term, so that a similar disc structure is maintained on reasonable time scales.

For the disc dynamo, the most important parameter is the dynamo coefficient $\alpha$ in the mean-field induction equation. The $\alpha$ effect is antisymmetric about the midplane and restricted to the disc. There is evidence that in accretion discs, where turbulence is driven by the magneto-rotational instability, $\alpha$ is negative in the upper disc half (Brandenburg et al., 1995; Ziegler and Rüdiger, 2000). A simple theoretical explanation is given in Brandenburg (1998), and a similar result has been obtained by Rüdiger and Pipin (2000) who considered magnetically driven turbulence; see also Rüdiger et al. (2001).

Is there any evidence that the $\alpha \Omega$ dynamo picture is correct and applicable to accretion discs? One piece of evidence is that the specific dependence of the large scale field geometry on the boundary conditions is the same both in simulations of shearing sheet turbulence driven by the magneto-rotational instability and in $\alpha \Omega$ dynamo models in the same geometry. Since the dynamo-generated magnetic field is of large scale and since it extends to the boundaries, one must expect a detailed dependence of its geometry on the boundary conditions. In discs with negative $\alpha$ in the upper half, with (pseudo-)vacuum boundary conditions one gets an oscillatory large scale field whose horizontal components are symmetric about the midplane. With perfect-conductor or finite-conductivity boundary conditions, one gets a non-oscillatory field whose horizontal components are antisymmetric about the midplane. The same is seen both in three-dimensional simulations and in solutions of the $\alpha \Omega$ dynamo problem; see Brandenburg (1998).

Bardou et al. (2001) found that vertical outflows do not change the symmetry or temporal behaviour of the dynamo-generated magnetic field. However, especially 
in the linear case for negative $\alpha$ in the upper disc half and finite-conductivity in the halo, an outflow tends to switch the disc dynamo off. This happens when the time scale of field stretching by the outflow becomes shorter than the regeneration time scale of the dynamo.

\section{Structured Outflow and Acceleration Mechanism}

Figure 2, left panel, illustrates that an outflow develops that has a well-pronounced structure. Within a conical shell originating from the inner edge of the disc, the terminal outflow speed exceeds $500 \mathrm{~km} \mathrm{~s}^{-1}$, and temperature and density are lower than elsewhere. The inner cone around the axis is the hottest and densest region where the stellar wind speed reaches about $150 \mathrm{~km} \mathrm{~s}^{-1}$. The wind that develops from the outer parts of the disc has intermediate values of the speed (see Figure 1).

The structured outflow is driven by a combination of different processes. A significant amount of angular momentum is transported outwards from the disc into the wind along magnetic field lines, especially along the strong lines within the conical shell (see Figure 2, right panel). The magnetic field geometry is such that the angle between the rotation axis and the field lines threading the disc exceeds
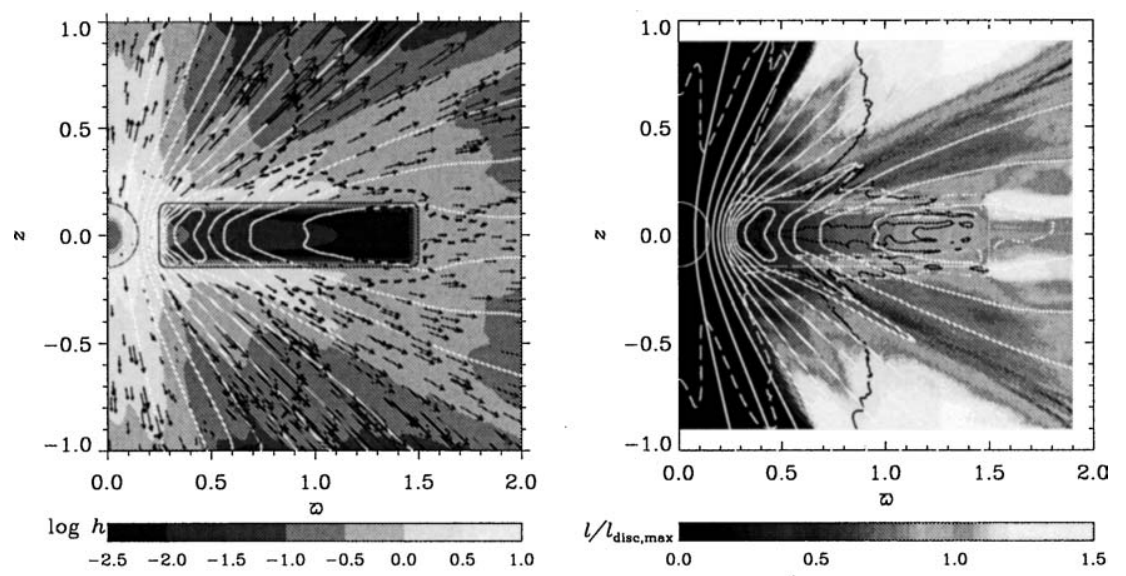

Figure 2. Left: poloidal velocity vectors and poloidal magnetic field lines (white) superimposed on a colour scale representation of $\log _{10} h$. Specific enthalpy $h$ is directly proportional to temperature $T$, and $\log _{10} h=(-2,-1,0,1)$ corresponds to $T \approx\left(3 \times 10^{3}, 3 \times 10^{4}, 3 \times 10^{5}, 3 \times 10^{6}\right) \mathrm{K}$. The black dashed line shows the fast magnetosonic surface. The disc boundary is shown with a thin black line, the stellar surface is marked in red. The dynamo $\alpha$ coefficient is negative in the upper disc half, resulting in roughly dipolar magnetic symmetry. Averaged over times $t \approx 897 \ldots 906$ days. Right: colour scale representation of the specific angular momentum, normalised by the maximum angular momentum in the disc, with poloidal magnetic field lines superimposed (white). The black solid line shows the Alfvén surface, the white dashed line the sonic surface. Same model as left and averaged over same times. 
$30^{\circ}$ at the disc surface, which is favourable for magneto-centrifugal acceleration (Blandford and Payne, 1982). However, the Alfvén surface is so close to the disc surface at the outer parts of the disc that acceleration there is mainly due to the gas-pressure force. In the conical shell, however, the outflow is highly supersonic but sub-Alfvénic, with the Alfvén radius a few times larger than the radius at the footpoint of the field lines at the disc surface. The lever arm of about 3 is sufficient for magneto-centrifugal acceleration to dominate in the conical shell (cf. Krasnopolsky et al., 1999). The inner cone has very low angular momentum and the magnetic lines are inclined to the axis by less than $30^{\circ}$ so that the stellar wind can only be gas-pressure driven. This picture is confirmed by looking at the ratio of magnetocentrifugal to gas-pressure forces, which is much larger than unity in the conical shell, where also the poloidal field lines are strongest. This is also the region where magnetic pressure produced by the toroidal field exceeds gas pressure, indicating that the magneto-centrifugally accelerated outflow in the conical shell is confined by the toroidal field.

\section{Magnetic Coupling Between Star and Disc}

Observations of T Tauri stars suggest that the stellar spin-down can be connected with a circumstellar accretion disc. Magnetic fields are believed to play an important rôle in the interaction between the star and its surrounding disc, resulting in particular in a spin-down of the star due to magnetic braking. Indeed, magnetic fields as strong as $10^{3}$ Gauss have been detected on T Tauri stars, and there is evidence for hot and cool spots on the stellar surface. Although the structure of the stellar magnetosphere is yet unknown, analytical models have been developed by Hartmann et al. (1994) and Shu et al. (1994) that assume that a stellar dipole threads a surrounding disc. In these static magnetospheric accretion models, disc matter is channelled along magnetospheric field lines to form a funnel-shaped polar accretion flow.

More realistic time dependent numerical simulations by Hirose et al. (1997) and Goodson et al. (1997) show episodic reconnection behaviour between a stellar dipolar magnetosphere and an imposed disc field, resulting in episodic mass transfer from the disc to the star. These authors find inner stellar jets and outer disc winds that are both driven by magneto-centrifugal processes.

Figure 3 shows our model with disc dynamo, where we add a stellar dipole that is anchored within the star, but can evolve with time outside the star (von Rekowski and Brandenburg, 2003). For the strong surface stellar field of about $6 \mathrm{kG}$ considered here, the time dependent accretion is correlated with the magnetic star-disc coupling. When the star is connected to the disc, accretion is along field lines (see Figure 3, left panel). When the star is disconnected from the disc, an enhanced wind carries away excess angular momentum (see Figure 3, right panel). The sum of magnetic, net accretion and viscous torques on the star is positive at radii between star and disc, which means a stellar spin-up. Spin-down torques only 

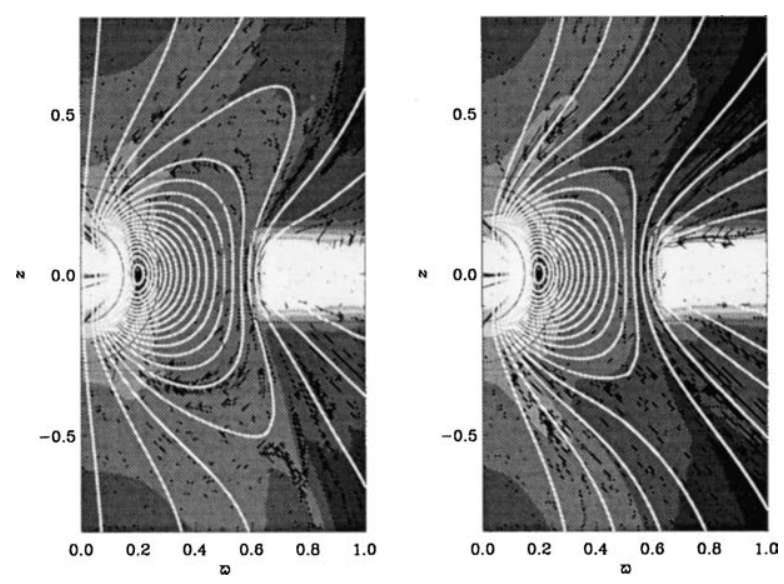

Figure 3. Colour scale representation of the density (bright colours indicate high values; dark colours, low values) with poloidal magnetic field lines superimposed (white) and the azimuthally integrated mass flux density shown with arrows (not shown in the disc). Left: at a time $t \approx 150$ days, when the star is magnetically connected to the disc. Right: at a time $t \approx 156$ days, when the star is disconnected from the disc.

result at latitudes where accretion takes place, because the angular velocity is very small between star and disc.

\section{Concluding Remarks}

Our simulations show that a system consisting of a magnetically coupled star and dynamo-active accretion disc can maintain a clearly structured outflow. The outflow consists of a (hotter and denser) slow stellar wind and a faster outer disc wind that are both gas-pressure driven, and a (cooler and less dense) fast magneto-centrifugally accelerated inner disc wind within a conical shell (see Figure 4, left panels). In addition, in transition periods, when the magnetic star-disc connectivity changes, there is a gas-pressure driven (small azimuthal velocity and angular momentum, the Alfvén surface close to the disc), hot and dense, but relatively fast outflow between the stellar and disc winds.

Coexisting gas-pressure and magneto-centrifugal driving mechanisms have also been found by Koide et al. (1998) who study the formation of relativistic jets from accretion discs surrounding a black hole by performing numerical simulations using a general relativistic magnetohydrodynamic code. In their model, a global (initially vertical) magnetic field penetrates the accretion disc which then leads to non-steady accretion and ejection processes. According to their simulations, a jet is ejected from the close vicinity of the black hole. The jet has a two-layered shell structure consisting of a fast gas-pressure driven jet in the inner part and a slow magnetically driven jet in the outer part, both of which are collimated by the global poloidal magnetic field penetrating the disc (see Figure 4, right panels). The fast jet is due 

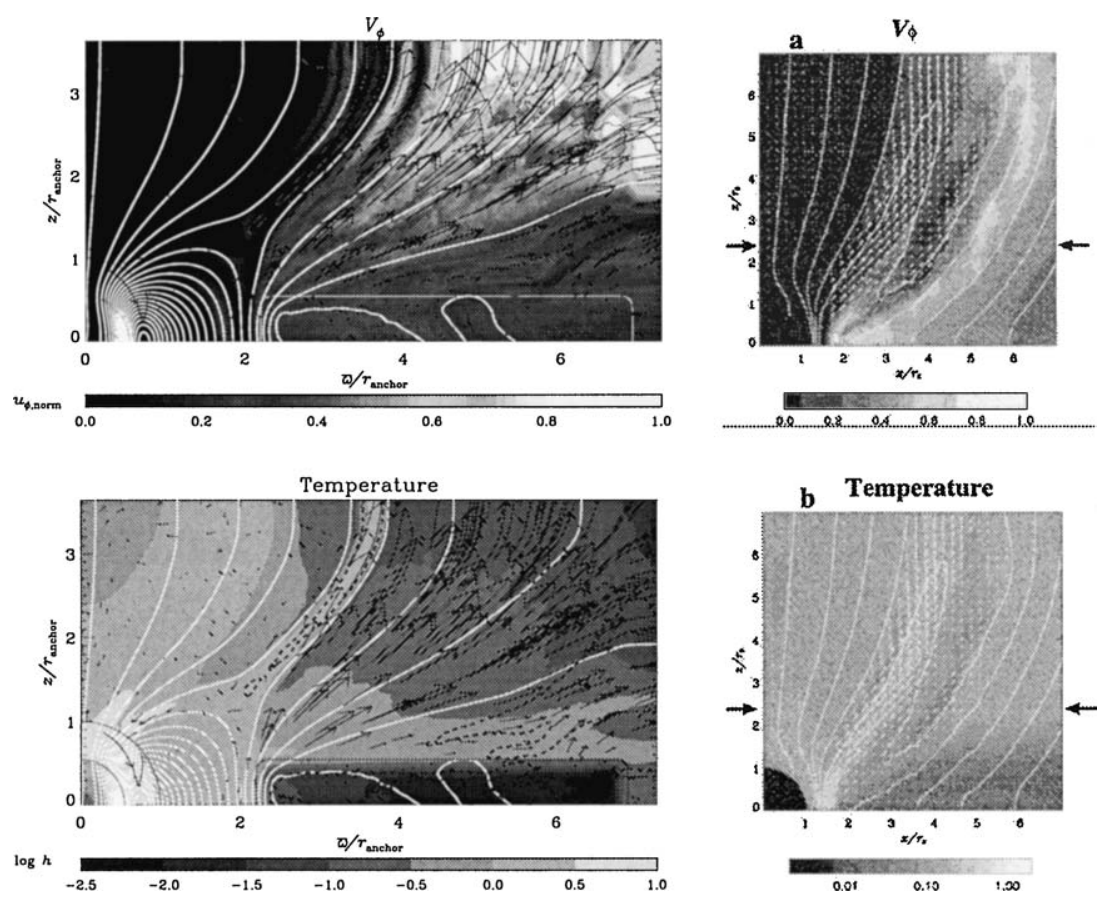

Figure 4. Colour scale representation of the normalised azimuthal velocity (top) and temperature (bottom) with poloidal magnetic field lines (white) and poloidal velocity vectors superimposed. Left: simulations of outflows from protostellar accretion discs by von Rekowski and Brandenburg (2003). Same model as in Figure 3, but at $t \approx 158$ days. Collimation is due to boundary conditions. Right: simulations of outflows from accretion discs around black holes by Koide et al. (1998).

to a strong gas-pressure increase due to shock formation at the inner edge of the disc, caused by a fast accretion flow.

Our relatively fast gas-pressure driven flow might be comparable with the fast jet. However, contrary to the slow jet, our magneto-centrifugally accelerated inner disc wind is even faster.

\section{Acknowledgements}

This work was supported by the PPARC Grant PPA/G/S/2000/00528. Use of the supercomputer SGI 3800 in Linköping and of the PPARC supported supercomputers in St Andrews and Leicester is acknowledged. This research was conducted using the resources of High Performance Computing Center North (HPC2N).

\section{References}

Bardou, A., von Rekowski, B., Dobler, W., Brandenburg, A. and Shukurov, A.: 2001, A\&A 370, 635. Blandford, R.D. and Payne, D.G.: 1982, MNRAS 199, 883. 
Brandenburg, A.: 1998, in: M.A. Abramowicz, G. Björnsson and J.E. Pringle (eds.), Theory of Black Hole Accretion Discs, Cambridge University Press, Cambridge, p. 61.

Brandenburg, A., Nordlund, Å., Stein, R.F. and Torkelsson, U.: 1995, ApJ 446, 741.

Goodson, A.P., Winglee, R.M. and Böhm, K.-H.: 1997, ApJ 489, 199.

Hartmann, L., Hewett, R. and Calvet, N.: 1994, ApJ 426, 669.

Hawley, J.F.: 2000, ApJ 528, 462.

Hirose, S., Uchida, Y., Shibata, K. and Matsumoto, R.: 1997, Publ. Astron. Soc. Japan 49, 193.

Koide, S., Shibata, K. and Kudoh, T.: 1998, ApJ 495, L63.

Krasnopolsky, R., Li, Z.-Y. and Blandford, R.: 1999, ApJ 526, 631.

Ouyed, R. and Pudritz, R.E.: 1997, ApJ 482, 712.

Ouyed, R., Pudritz, R.E. and Stone, J.M.: 1997, Nat 385, 409.

Reyes-Ruiz, M. and Stepinski, T.F.: 1995, ApJ 438, 750.

Rüdiger, G. and Pipin, V.V.: 2000, $A \& A$ 362, 756.

Rüdiger, G., Pipin, V.V. and Belvedère, G.: 2001, Sol. Phys. 198, 241.

Shu, F., Najita, J., Ostriker, E., Wilkin, F., Ruden, S. and Lizano, S.: 1994, ApJ 429, 781.

von Rekowski, B. and Brandenburg, A.: 2004, $A \& A$ 420, 17.

von Rekowski, B., Brandenburg, A., Dobler, W. and Shukurov, A.: 2003, A\&A 398, 825.

Ziegler, U. and Rüdiger, G.: 2000, A\&A 356, 1141. 